\title{
A Novel Optimized Golomb-Rice Technique for the Reconstruction in Lossless Compression of Digital Images
}

\author{
Shaik. Mahaboob Basha ${ }^{1,2}$ and B. C. Jinaga ${ }^{1}$ \\ ${ }^{1}$ Department of E.C.E., J.N.T. University, Hyderabad, Andhra Pradesh 500085, India \\ ${ }^{2}$ Department of E.C.E, Priyadarshini College of Engineering \& Technology, Nellore, Andhra Pradesh 524004, India
}

Correspondence should be addressed to Shaik. Mahaboob Basha; mohisin7@yahoo.co.in

Received 25 June 2013; Accepted 17 July 2013

Academic Editors: I. Guler and S. Kalitzin

Copyright (c) 2013 Shaik. M. Basha and B. C. Jinaga. This is an open access article distributed under the Creative Commons Attribution License, which permits unrestricted use, distribution, and reproduction in any medium, provided the original work is properly cited.

\begin{abstract}
The research trends that are available in the area of image compression for various imaging applications are not adequate for some of the applications. These applications require good visual quality in processing. In general the tradeoff between compression efficiency and picture quality is the most important parameter to validate the work. The existing algorithms for still image compression were developed by considering the compression efficiency parameter by giving least importance to the visual quality in processing. Hence, we proposed a novel lossless image compression algorithm based on Golomb-Rice coding which was efficiently suited for various types of digital images. Thus, in this work, we specifically address the following problem that is to maintain the compression ratio for better visual quality in the reconstruction and considerable gain in the values of peak signal-to-noise ratios (PSNR). We considered medical images, satellite extracted images, and natural images for the inspection and proposed a novel technique to increase the visual quality of the reconstructed image.
\end{abstract}

\section{Introduction}

Digital images have become very common in the present societal needs, especially in the electronic industry and other applications like medical imaging, satellite imaging, multimedia applications, and high-speed data internet data paths. The concept of image processing thus leads to the evolution of different image acquisition devices like still and video cameras and web cameras. Thus, our work is to designate a novel technique for solving the forward as well as inverse problems in the still image compression. The solutions derived from these forward and inverse matrix computations facilitated a comprehensive meaning for the image compression for JPEG 2000 and JPEG-LS [1-3].

\section{Review of Existing Techniques}

JPEG 2000 still image compression standard supports both lossy and lossless compression schemes, whereas JPEG-LS is a lossless image compression scheme. The JPEG-LS uses statistical based coding techniques such as Golomb, Huffman coding, and Golomb-Rice (GR) coding techniques. Nowadays most of the VLSI-based companies and multimedia application oriented industries are offering the Golomb and Golomb-Rice coding for their applications. Thus, it is observed from the literature survey that the necessity of optimization is an essential parameter in image compression.

Somasundaram and Sumithra [4] derived a compression algorithm using the GC technique. In their research work it was shown that the results were compared with existing coding techniques along with the standard EBCOT. The maximum PSNR achieved is $68 \mathrm{~dB}$. The work was executed for bpp values ranging from 0.01 to $0.75 \mathrm{bpp}$. Meany and Martens [5] derived a technique in this area. The algorithm uses split field coding to attain less complexity.

The development of different techniques for the images of various types of domains is the critical issue in the research area. Thus, we investigated and developed a lossless 
image compression termed as a novel coding technique. The reconstruction of the image resembles with the original input image at the receiving side with good visual quality.

Golomb coding invented by Solomon Golomb emerged as an important tool in lossless compression. The well-known coding technique in the area of lossless image compression is embedded block coding with optimal truncation (EBCOT). Due to its limitations regarding computation time and image sizes the Golomb-Rice coding is considered as the replacement for EBCOT. After that the DCT compression using the GR coding technique proposed by Kadayam Thyagarajan becomes an interesting look towards lossless image compression. We considered DWT highly reduces the redundancy than DCT.

From the basics of the GR code, the nonnegative integer of each code is to be extracted first. For the encoding process the extracted nonnegative integer " $i$ " and the rank " $k$ " are to be considered. Then, we encoded the code word prefix that is $\left\lfloor i / 2^{k}\right\rfloor$ using unary code. Here the suffix is $i$ $\bmod 2^{k}$ using $k$-bit natural binary code [6]. The Golomb parameter, quotient, and run length at zero condition are derived. The concatenation process is elevated for remainders and quotients.

\section{Role of Scalar Quantization in the Proposed Work}

Among the two quantization techniques, that is, vector and scalar, the scalar quantization is essential for arranging the DWT coefficients in the prescribed set of amplitude levels. In general, either uniform or non uniform quantization can be performed. But the preferable is nonuniform for lossy compression techniques because of its nature of variation from one static subband image to another. The static image is then reconstructed using the general decompression process with multiresolution analysis of level 2 , and the reconstructed image was displayed for performance measure.

\section{Flow Chart of the Proposed Novel Algorithm}

We investigated here the optimization techniques for a good reversible DWT decomposed image through the proposed technique. Various types of images were selected for simulation to measure the performance levels of the technique. The input image is grouped in blocks of $4 * 4$ pixels to minimize the computational complexity. The quotient and remainder values are preserved from each encode step.

By selecting the optimum compression technique, the flexibility was achieved for the requirement of various specified applications in the work. The discrete wavelet transform (DWT) family embedded is biorthogonal 3/5. This biorthogonal 3/5 helps in the perfect reversible process [7]. The filter taps are having 3 and 5 filter coefficients for LPF and HPF designs, respectively [8].

The discrete meyer (DMEY) wavelet efficiently worked [8] and has given better results with the proposed technique. The compression and reconstruction results are studied and

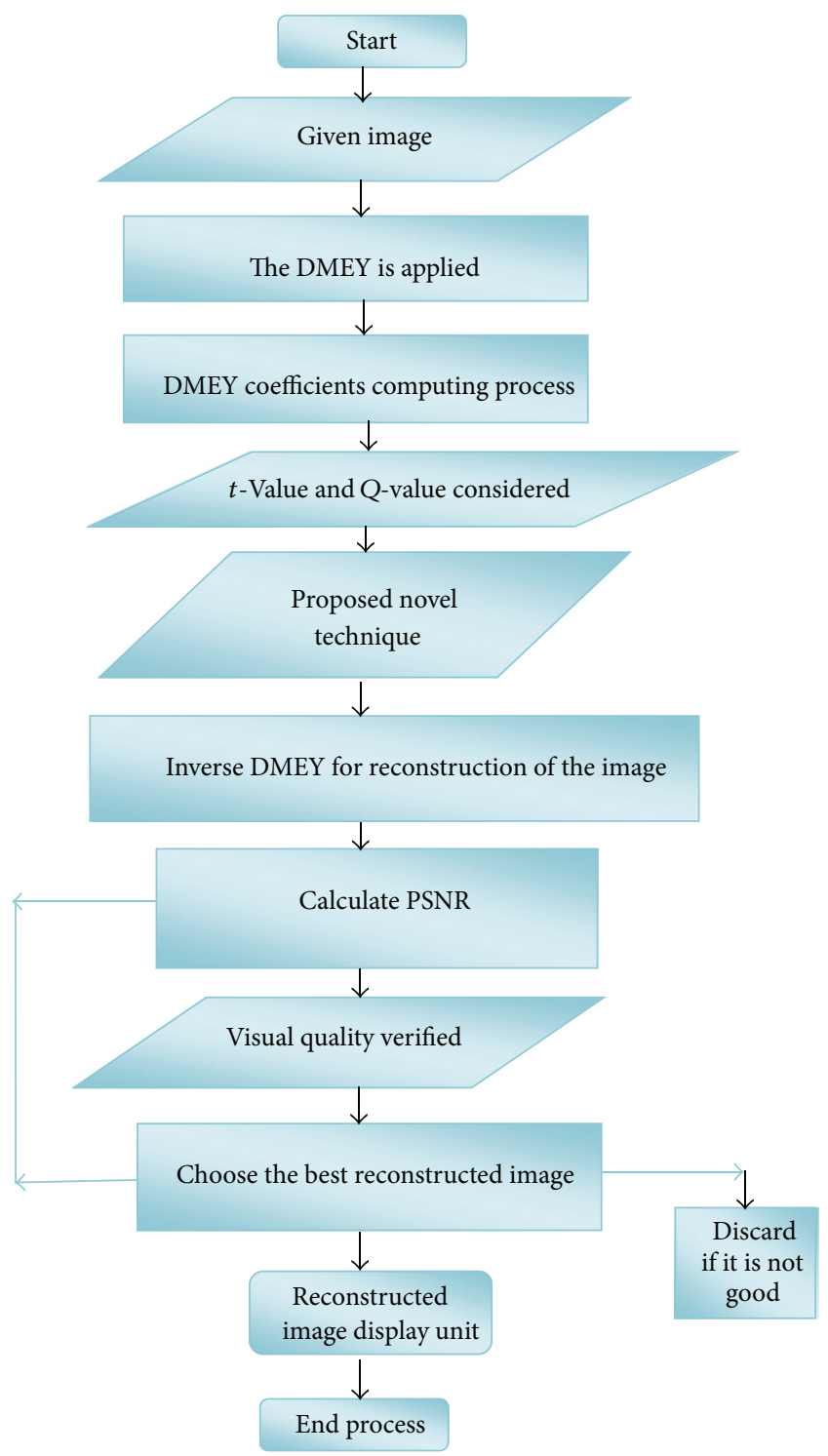

Figure 1: The flow chart of the proposed coding.

evaluation done to show the comparative better performance with existing methods. Due to its reversible nature, the DMEY is incorporated in the developed algorithm. We selected level 2 as it gives a better approximation of the original image. The flow chart of the proposed technique is shown in Figure 1.

\section{Proposed Coding Procedure}

The procedure of developed coding is explained in the following section.

Step 1. The parameter has to be fixed to noninteger value.

Step 2. Generating of the code word by encoder.

Step 3. Select unary coding for quotient and truncated binary encoding for the reminder as per GR procedure. 
Step 4. For GR code $\log _{2}(M)$ bits are necessary, and they can be loaded.

Step 5. Bit reversal order of given data is applied.

Step 6. Rearrange the new data into two groups G1 and G2.

Step 7. Flip the G1 bits and transmit after the conversion into Gray code.

Step 8. Flip the G2 bits and transmit after the conversion into Gray code.

Step 9. Then the data is sent through the selected transmitting medium.

Step 10. End.

We proposed a novel technique in the direction to measure the performance by considering the relationship between the Q-value and PSNR values. Thus, the developed algorithms were compared with the present valuable contributions mentioned in the references [4, 9]. Simulated graphs are incorporated as Figures 5, 6, and 7.

We considered image pixels of 8 bits. The quantized version of the DWT transformed coefficients, especially LL band, and the quotient, the remainder derived from the GR coding, were taken as inputs for the proposed novel algorithm. The proposed algorithm begins with a bit reversal of all transformed pixel values.

Then the encoded data bits are made into two groups, namely, G1 and G2. At first, G2 bits are inverted. After that the inverted G2 bits are encoded using Gray code to allow data bits to transmit; that is, the output bits are allowed to any channel for transmission. The channels may be wired or wireless. The condition considered is that the DWT level 2 is applied for the image to decompose it into subbands. For the perfect reversible process, biorthogonal $3 / 5$ is used. The developed technique exhibits better results than the existing and is well suited for the medical, satellite, and natural images. The computational cost and complexity can be considerably reduced using the proposed technique if it is implemented on VLSI validation.

\section{Simulation Results}

The proposed novel technique was tested on different image sizes, and the results are incorporated in Tables 1, 2, and 3. We applied and tested the proposed algorithm on different images. Quantitative results were posted in tables for some images, and the processed images are shown for remaining tested images.

The general formulae of bits per pixel and PSNR are given below. The performance of the algorithm was estimated with respect to PSNR value and visual quality:

bits per pixel (bpp)

$$
=\left(\frac{\text { Output image size }}{\text { Input image size }}\right) \times 8 \text {. }
$$
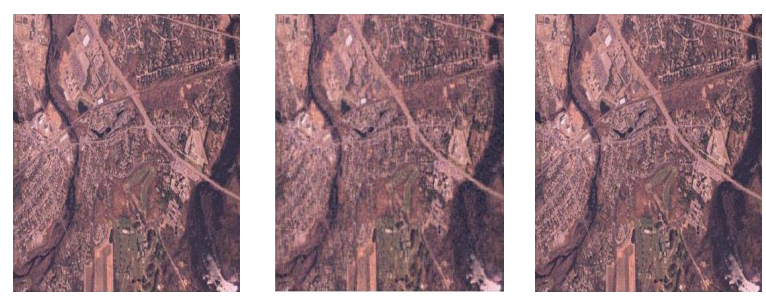

FIGURE 2: The original, compressed, and decompressed images of aerial.
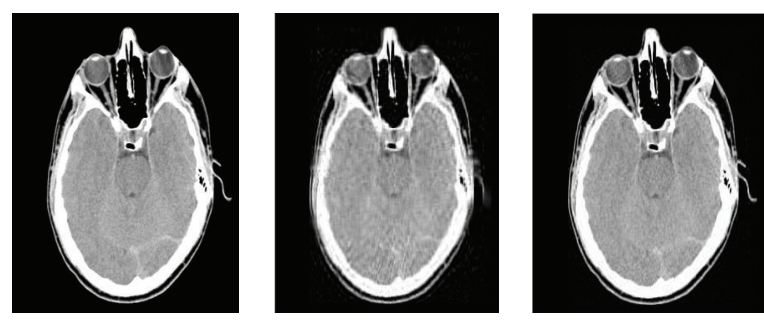

FIGURE 3: The original, compressed, and decompressed images of head.

The image sizes are measured in bytes:

peak signal-to-noise ratio

$$
=\mathrm{PSNR}=20 \log 10\left(\frac{255}{\sqrt{\mathrm{MSE}}}\right),
$$

where MSE $=$ mean square error.

6.1. Description of Example (Aerial). We applied the proposed novel algorithm on the aerial image. The DMEY decomposes the image to the second level using reversible wavelet family, and the DMEY coefficient version of the image is given as inputs to the proposed algorithm. The compressed image and the decompressed image are shown in Figure 2. It is observed that the reconstructed image is near the appearance of the original image. Thus, the objective of research work is achieved.

Now, the resulting images are studied and viewed at different values of $Q$.

At $Q=4$, the image had better compression ratio and poor PSNR value [8].

At $Q=12$, image quality is better than 4 but there is reduction in compression ratio.

Finally, at $Q=34$, the PSNR value reaches highest and the good visual image is noticed. Thus, the aim of the work is achieved.

The $Q$-value range even in this case is also considered as 4 to 34 which in turn affects the compression ratio. Similarly, it is noticed that in all the cases at the $Q$-value of 34 the image appeared in good quality with better compression ratio. The key observation is that the derived PSNR values showed slight variations depending upon the chosen images (Figures 3 and 4).

The derived values are incorporated in Tables 1, 2, and 3 . 
TABle 1: Performance comparison results with existing techniques (chest).

\begin{tabular}{|c|c|c|c|c|c|c|}
\hline \multirow{2}{*}{ Bits per pixel (bpp) } & \multicolumn{3}{|c|}{ PSNR in $\mathrm{dB}$ at $Q=0$} & \multicolumn{3}{|c|}{ PSNR in $\mathrm{dB}$ at $Q=34$} \\
\hline & Reference [9] & Reference [4] & Proposed algorithm & Reference [9] & Reference [4] & Proposed algorithm \\
\hline 0.01 & 28.14 & 29.09 & 31.18 & 28.32 & 28.92 & 32.85 \\
\hline 0.20 & 35.68 & 36.13 & 38.04 & 36.10 & 36.92 & 39.16 \\
\hline 0.40 & 42.80 & 43.72 & 45.16 & 43.22 & 43.39 & 47.06 \\
\hline 0.60 & 52.06 & 52.95 & 54.08 & 52.06 & 52.41 & 56.12 \\
\hline 0.75 & 67.71 & 67.91 & 69.24 & 67.71 & 68.31 & 70.46 \\
\hline 0.80 & 68.56 & 69.71 & 71.56 & 68.68 & 69.35 & 72.56 \\
\hline
\end{tabular}

TABLE 2: Performance comparison results with existing techniques (peppers).

\begin{tabular}{lcccccc}
\hline \multirow{2}{*}{ Bits per pixel (bpp) } & \multicolumn{3}{c}{ PSNR in $\mathrm{dB}$ at $Q=0$} & \multicolumn{3}{c}{ PSNR in $\mathrm{dB}$ at $Q=34$} \\
& Reference [9] & Reference [4] & Proposed algorithm & Reference [9] & Reference [4] & Proposed algorithm \\
\hline 0.01 & 20.9 & 21.83 & 23.16 & 21.04 & 22.03 & 24.18 \\
0.20 & 31.2 & 32.13 & 34.58 & 31.66 & 32.65 & 36.52 \\
0.40 & 38.02 & 38.97 & 40.67 & 39.63 & 40.62 & 42.68 \\
0.60 & 46.2 & 47.13 & 49.02 & 47.3 & 48.29 & 49.98 \\
0.75 & 63.87 & 64.85 & 67.01 & 63.87 & 64.21 & 69.64 \\
0.80 & 64.78 & 66.21 & 69.78 & 64.67 & 65.54 & 70.54 \\
\hline
\end{tabular}
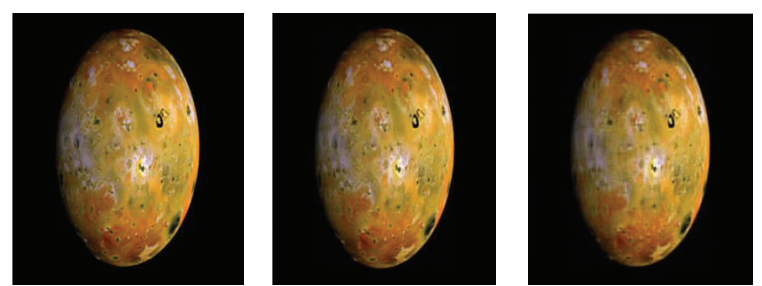

FIGURE 4: The original, compressed, and decompressed images of Jupiter.

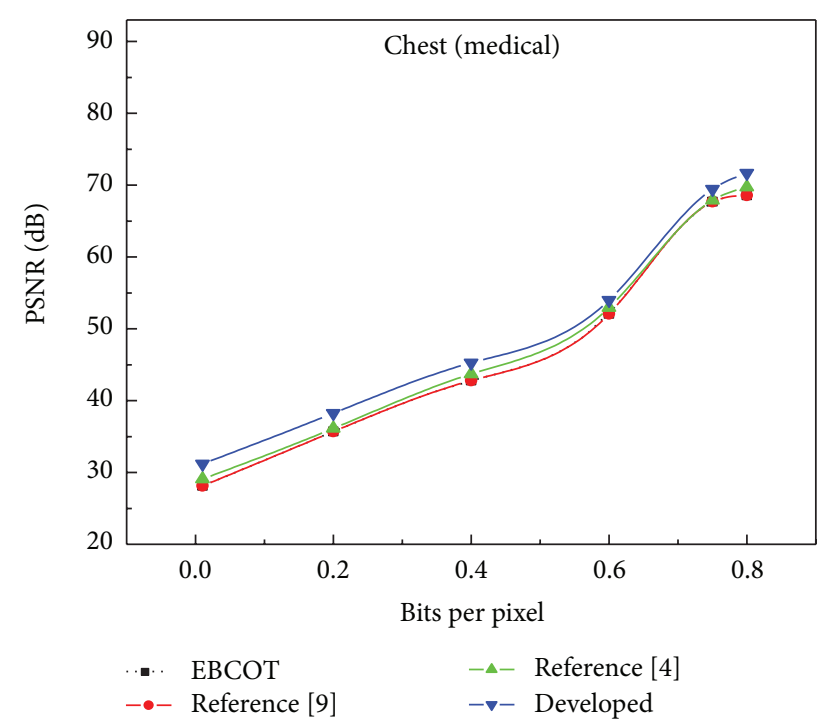

FIGURE 5: Comparison of developed versus existing techniques (chest).

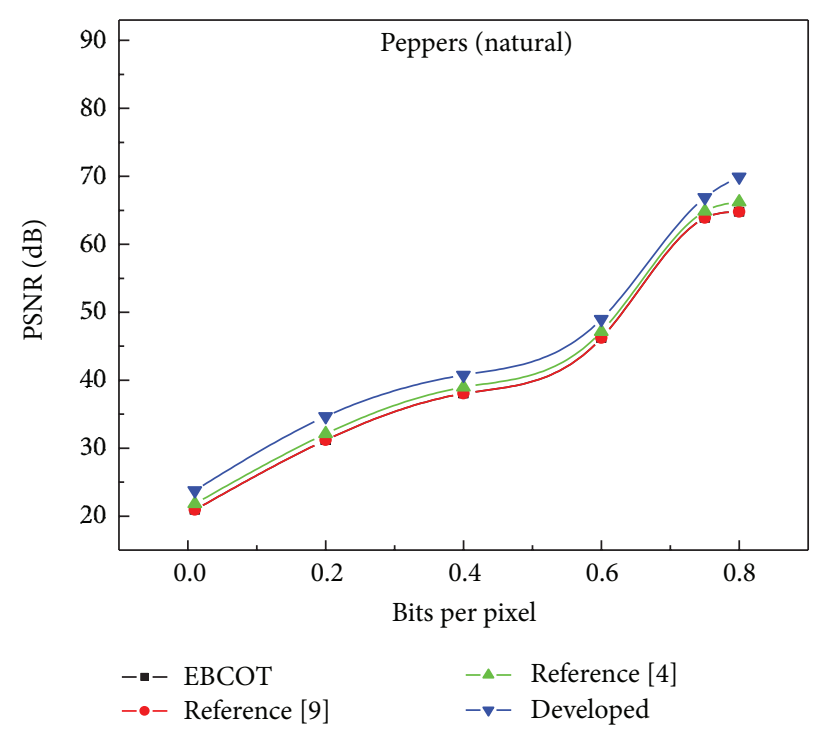

FIGURE 6: Comparison of developed versus existing techniques (peppers).

\section{Conclusion and Future Scope}

The developed novel technique for the lossless image compression technique investigated the bottlenecks of the existing algorithms. The visual quality of the compressed images and the reconstructed images seems to be good by maintaining the compression efficiency high. The proposed technique shows better results when compared with existing methods. The important parameter in the compression, that is, the PSNR value, is highlighted, thus resulting in good processed 
TABle 3: Performance comparison results with existing techniques (Jupiter).

\begin{tabular}{|c|c|c|c|c|c|c|}
\hline \multirow{2}{*}{ Bits per pixel (bpp) } & \multicolumn{3}{|c|}{ PSNR in $\mathrm{dB}$ at $Q=0$} & \multicolumn{3}{|c|}{ PSNR in $\mathrm{dB}$ at $Q=34$} \\
\hline & Reference [9] & Reference [4] & Proposed algorithm & Reference [9] & Reference [4] & Proposed algorithm \\
\hline 0.01 & 19.48 & 20.4 & 21.87 & 19.53 & 20.46 & 23.04 \\
\hline 0.20 & 26.26 & 27.21 & 28.48 & 26.86 & 27.79 & 30.06 \\
\hline 0.40 & 32.96 & 33.95 & 36.01 & 35.52 & 36.47 & 38.14 \\
\hline 0.60 & 41.45 & 42.44 & 44.56 & 43.85 & 44.81 & 46.86 \\
\hline 0.75 & 48.89 & 49.88 & 51.78 & 50.39 & 51.38 & 53.98 \\
\hline 0.80 & 50.64 & 52.06 & 54.04 & 51.32 & 52.04 & 56.16 \\
\hline
\end{tabular}

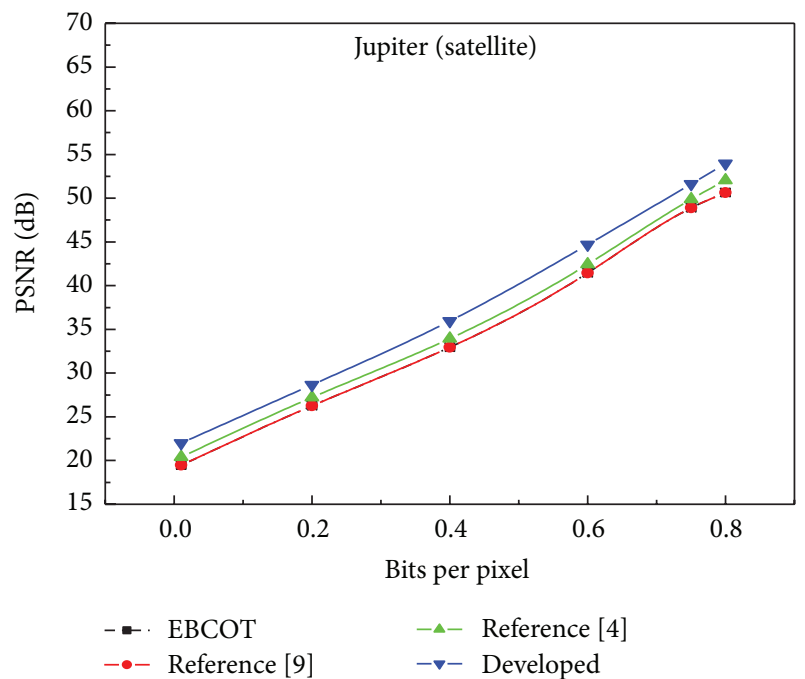

FIGURE 7: Comparison of developed versus existing techniques (Jupiter).

images. The proposed algorithm is executed on different images such as medical images, satellite images, and natural images like Lena for the high visual quality. Further, the validation for natural images can also be carried out.

\section{Acknowledgment}

Shaik. Mahaboob Basha feels profoundly indebted to his research supervisor Dr. B. C. Jinaga for the opportunity to work with him and benefit from his valuable guidance and knowledge. It gives him immense pleasure to express his deep sense of gratitude to his research supervisor and guide Dr. B. C. Jinaga for rendering his inestimable help, encouragements, and constructive suggestions at every stage of his research work.

\section{References}

[1] R. F. Rice and P. J. Plaunt Jr., "Adaptive variable-length coding for efficient compression of spacecraft television data," IEEE Transactions on Communications, vol. 19, no. 6, pp. 889-897, 1971.

[2] S. W. Golomb, "Run-length encodings," IEEE Transactions on Information Theory, vol. 12, no. 3, pp. 399-401, 1966.
[3] L. Lu and S. Deng, "Study on JPEG2000 optimized compression algorithm for remote sensing image," in Proceedings of the International Conference on Networks Security, Wireless Communications and Trusted Computing (NSWCTC '09), vol. 2, pp. 771-775, IEEE Computer Society, Wuhan, China, April 2009.

[4] K. Somasundaram and P. Sumithra, "A Novel method to compress still images using Golomb code (GC) in JPEG2000," International Journal of Complete Science and Network Security, vol. 10, no. 8, pp. 182-186, 2010.

[5] J. J. Meany and C. J. Martens, "Split field coding: low complexity error-resilient entropy coding for image compression," in Applications of Digital Image Processing XXXI, vol. 7073 of Proceedings of SPIE, San Diego, Calif, USA, August 2008.

[6] M. A. Mofaddel and W. M. Abd-Elhafiez, "Object-based hybrid image and video coding scheme," in Proceedings of the International Conference on Computer Engineering and Systems (ICCES'11), pp. 245-251, Cairo, Egypt, December 2011.

[7] M. S. Dawood, L. Ahila, and S. Sadasivam, "Image compression in wireless sensor networks-a survey," International Journal of Applied Information Systems, vol. 1, no. 9, pp. 11-15, 2012.

[8] S. M. Basha and B. C. Jinaga, "A Robust image compression algorithm using JPEG2000 standard with Golomb Rice coding," International Journal of Computer Science and Network Security, vol. 10, no. 12, pp. 26-33, 2010.

[9] T. Sanguankotchakorn and J. Fangtham, "A new approach to reduce encoding time in EBCOT algorithm for JPEG2000," in Proceedings of the IEEE Conference on Convergent Technologies for the Asia-Pacific Region (TENCON '03), vol. 4, pp. 1338-1342, Bangalore, India, October 2003. 

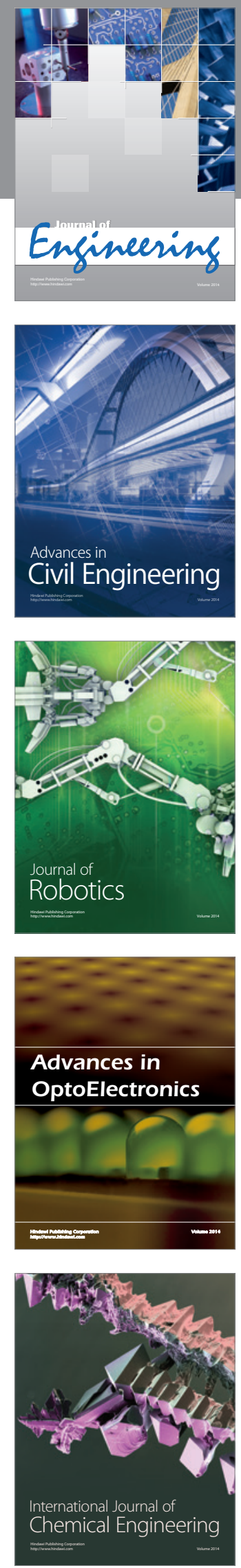

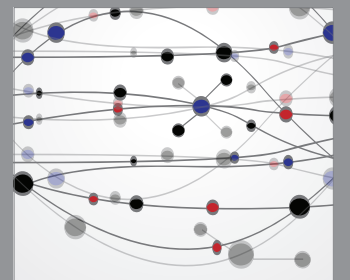

The Scientific World Journal
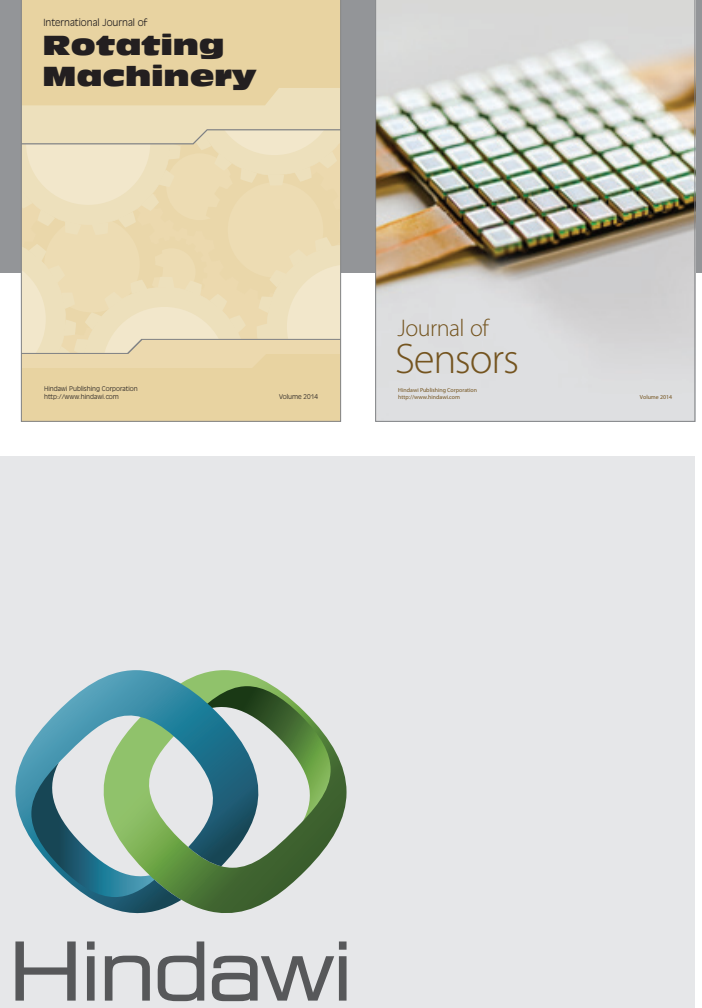

Submit your manuscripts at http://www.hindawi.com
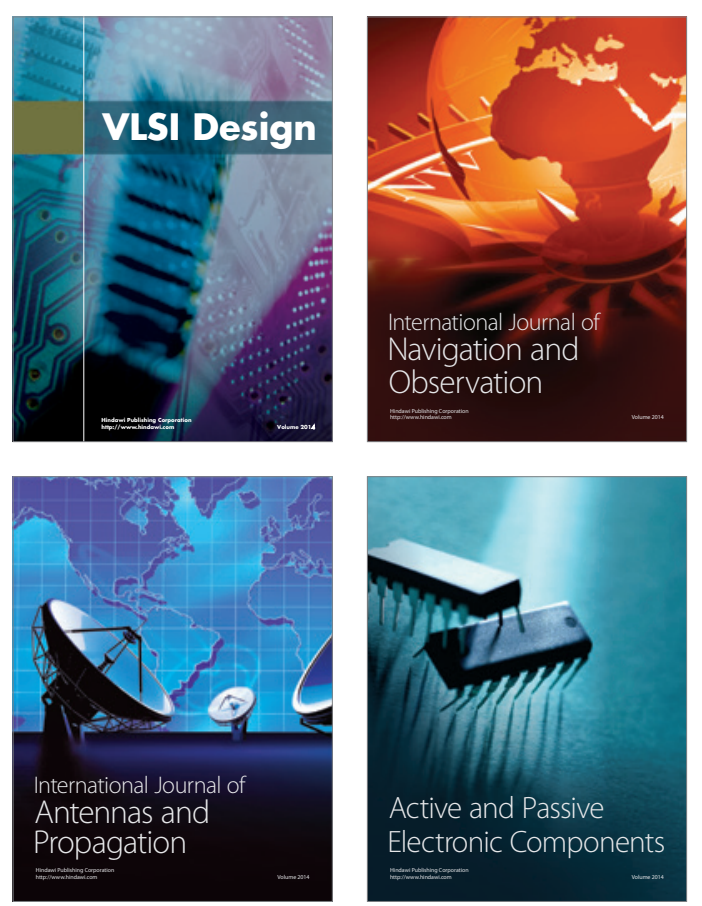
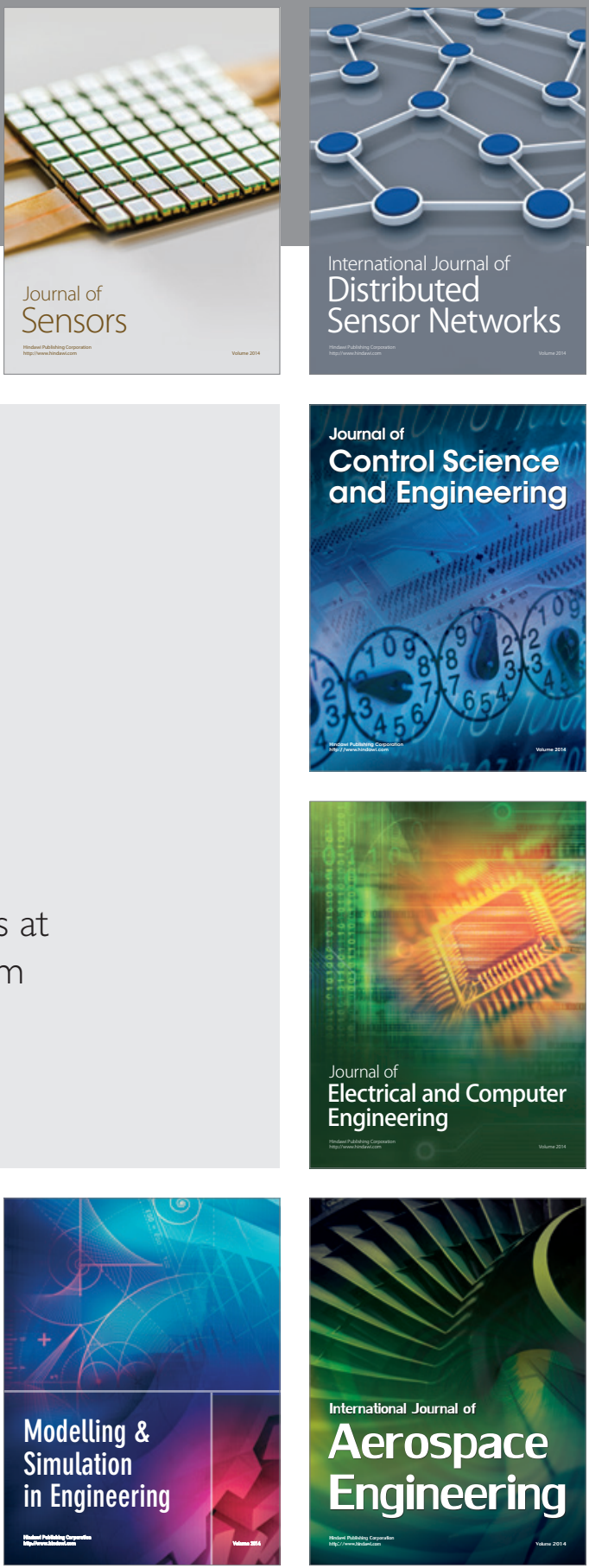

Journal of

Control Science

and Engineering
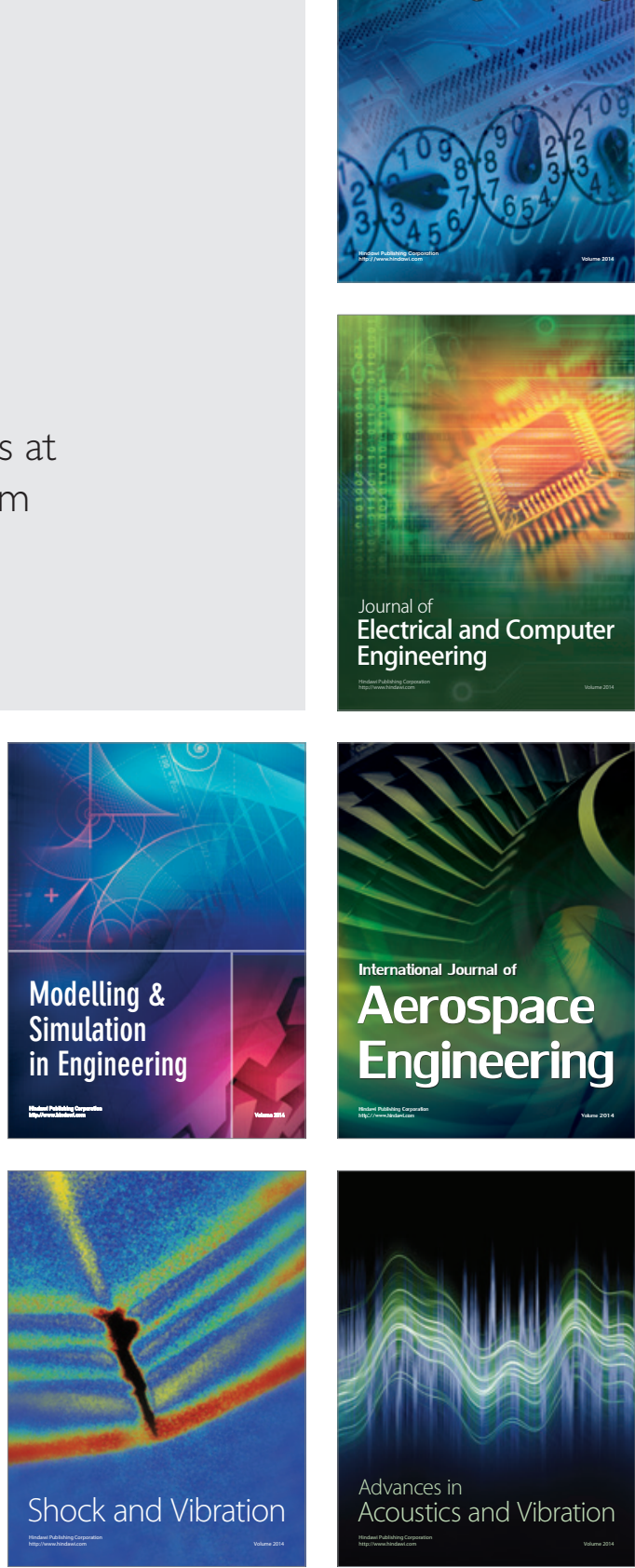